\title{
Application of Clothing Accessories in Clothing Display Design
}

\author{
Rui Guo \\ College of Art and Appareluages, Tianjin Polytechnic University, Tianjin 300160, China \\ E-mail: mypace008@yahoo.com.cn
}

\begin{abstract}
Clothing accessories also known as clothing decoration, such as hats, scarves, shoes and socks, etc. It directly affects the show results of clothing display design. This paper analyzes the application of clothing accessories in clothing display design from creating atmosphere of dress and disseminating brand culture, tries to raise the level of applying clothing accessories to clothing display design.
\end{abstract}

Keywords: Clothing design display, Clothing accessories, Use

\section{Clothing accessories and clothing display design}

\subsection{Overview of Clothing accessories and clothing display design}

Clothing accessories, also known as clothing decoration, are all accessories to complete dress state except garment, including ornaments in head, neck, chest, waist, wrist, fingers and feet.(1,Liu Xiaogang ,2005,P.50)Clothing accessories are closely related to the development of human society. According to archaeological discoveries, clothing accessories appear as early as clothing. It is divided into two major categories: practical accessories, decorative accessories. No matter what the era is, it seems that people prefer clothing accessories to clothing. Clothing accessories is indispensable when matching clothing, with the contents of clothing, especially in the modern life. With the continuous development of the fashion industry, the practicability and ornamental characteristic of clothing accessories are gradually merging into each other, their boundaries are becoming blurred increasingly.

Today, Fashion display design has become professional disciplines by the combination of visual art, space science and marketing strategy.(2, Qu Wenbo,1999,P.76).It is a comprehensive multi-discipline of marketing, aesthetic, consumer psychology and visual arts. It is explained in English: Display, Visual Presentation or Visual Merchandising Presentation. As an integral part of fashion design and planning, clothing display design is an action of display around clothing and brand. In the process of clothing display design, designers decorate and arrange products with showcase, shelf, prop, lighting, poster and other forms to display overall dress style, enhance brand image, communicate cultural connotation.

\subsection{Accessories component in the display clothing goods}

\subsubsection{Head Accessories}

Head accessories refer to the accessories used in thehead, such as hair card, hair hoop, hat, glasses and earrings. The most representative one is hat. Wide varieties of hats have different shapes and textures which have practical and decorative function. According to styles of clothing matching, it can be divided into various hats like leisure hat, fashion hat and topper.

\subsubsection{Upper Body Accessories}

This clothing accessories wearing in the upper part of the body are collectively referred to as "upper body accessories". These varieties of accessories include the most things, such as scarf, shawl, necklace, brooch, gloves, bracelet and finger decoration. The part of the body above the chest is vision center, so these upper body accessories have strong sense of decoration. In particular, it should be pointed out that bag, classified as upper body accessories, is used most extensive in clothing display design, which has the most practicality and decorative characteristic.

\subsubsection{Lower Body Accessories}

Corresponding to upper body accessories, the lower body accessories mainly include belt, shoes and socks. These parts of accessories have more practicality, which are not only looked beautiful, but also felt comfortable. They reflect the integrity of clothing very well.

\subsection{The purpose of use of accessories in clothing display design}

Through shaping, coloring and ornamenting, clothing accessories compensate for the lack of clothing, use their unique artistic language to create different atmosphere of life dress and meet psychological needs of different people, and 
become a very important decorative component in clothing display design.

The application of clothing accessories in clothing displayed design can achieve the following results: (1) highlighting the integrity of clothing to improve the grade and quality of clothing. It can sum up and upgrade clothing design ploy from the single and limited state in the past. Through the distinctiveness of style and the integrity of matching, the beauty of clothing style obtains further distillation and minimizes the homogenization of products.(2) Guiding consumption trends, meeting various needs. Because of sheep mentality, consumers want to avoid too duplicate and outdated in the circumstances that roughly the same style can be ensured. Therefore, clothing accessories provide more choice of space for them. This not only meets the needs of different consumers for highlighting personality, but also leads the fashion trend of consumption.(3) Enhancing the visual marketing, communicating brand concept. The unified, coordinated and integrated image of apparel products is not only conducive to the overall layout of goods, but also to provide a good shopping environment for consumers. It conveys the integrated image of brand, at the same time completes the "AIAD" (Attention, Interest, Desire and Action) visual marketing. (3, Ma Dali, 2006, P109).Achieving economic efficiency eventually.

\section{Application of clothing accessories in clothing display design}

\subsection{Create an atmosphere of dressing}

Chanel (CoCo Chanel), fashion design master, believes that:" Accessories are the tool not for maintaining value, but for forming the best means of visual effects when matching fashion."(4, Wang Shouzhi, 2002, P.69).

Because of the dependent and subordinate characteristics in clothing design, clothing accessories echo the popular theme of clothing display to form a unified fascinating visual effect when they are designed in shape, color and material aspects.

For clothing accessories, color should be used moderately, and then certain ratios will bring a holistic beauty.(5, Jia Jingsheng,1999,P.256).It could have an adverse effect if the color of clothing accessories were to be over-exaggerated or waked excessively. The three principles of color, based on similar color, analog color and contrast color, cause the relationship of contrast, harmony, stress and echo formed between accessories and clothing, and add the sense of richness and hierarchy for clothing display design.

In the aspect of shaping, matching of clothing accessories should follow the principle of composition of point, line and surface to form the formal beauty which is orderly, rhythmical, focused and coordinated. Guess what effect would be if skilful occupational apparel matched a pair of round toe shoes? The answer may be that the unified and harmonious formal beauty be ruined by the two different style of shaping.

Accessories' material generally refers to the integrative sense of seeing and feeling formed by the character, texture, color and luster of raw materials. Leather feels stiff which shows capable and spiffy; silk feels smooth which shows elegant and feminine; plush feels soft which shows easeful and cozy. Women's femininity would be displayed thoroughly if a soft plush coat matched a smooth scarf.

In clothing display design, accessories are visual information symbols which can not be ignored, play improved and reinforced role in highlighting display theme. Clothing accessories should match clothing coordinately and orderly based on the following aspects of apparel goods: pattern, color, style, quality and price. The themes of display design are different; even then the duplicate accessories, like shoes, socks, hat, bag, belt, can be chosen in matching women's wear; men's wear also can use the similar accessories, including shirt, tie and belt, to match different types of suit as complete set of product series for displaying. Consumers prefer imagining the overall effect of dress, so integrated matching makes consumer more intuitive and effective to contrast and choose, then related sale become truth. The aspects that clothing fall short of in display design, clothing accessories can make up defect and shortage; further, they can play a role in strengthening dress effect. But when using accessories to create dress styles, designers do not blindly pursue the expressive force of pure art and ignore the flexibility and fashion of business performance.

\subsection{Spread of brand culture}

As one of the means of enterprise marketing, clothing accessories could not match clothing simply in display design; as a result, the inherent requirements of brand culture were ignored. It is contrary to the final object that "display is not only selling goods, but also making the brand enjoys popular support". Generally speaking, the selection and application of accessories should serve brand culture absolutely in display. In color, shape and styles, CHANEL's accessories, like shoes, bags, glasses, ornaments, and DIOR's, like underwear, perfume, cosmetics, jewelry, fur, gloves, socks, formed the uniform of visual effect with apparel products. The uniform of visual effect helped clothing achieve the integrity of matching, at the same time showed brand features and spread brand culture.

The well-known clothing brands have being established by long usage to show their culture connotation. JUDGER, for instance, is known for its elegant suits, just like it's advertising:" Dressing solemnly, living auspiciously". If casual hats and shoes were appeared in display design, which would mean that JUDGER is conveying the information: there are 
casual wear in its series of products. Therefore, the features of casual display will be considered either. The major apparel products of METERSBONWE are sports wear and casual wear. Using sun hat, sports cuff, climbing shoes and traveling bag in display design, the happy scenario of young people in summer travel is formed, which vividly provide an immersive space of imagination for consumers. Like the black and white of ADIDAS, the pink of FAIRYFAIR, accessories can match based on the color of brand image. The application of accessories should not be realistic, but be notional, which should convey the information that its brand culture will be a fashion guide in the future to lead consumers to long for the fashion and pursue it.

In short, the application of clothing accessories makes clothing display design more meaningful, makes brand more popular, makes consumer more loyal.

\section{Concluding Remark}

To sum up, clothing and accessories are interdependence of the two aspects, they are independent but interactive. The application of accessories in clothing display design is not immutable, but changeable. It should constantly bring freshness to consumers. In clothing display design, the appropriate match of accessories is not only create the image of dress code belong to different styles, but also increase the value-added of apparel products to lead clothing enterprise out of its predicament of products homogenization and enhance the market competitiveness; there by, through artistic displaying, it makes apparel products realize commercial value.

\section{References}

Liu, Xiaogang. (2005).Fashion design art. Shanghai: Donghua University Press.

Qu, Wenbo. (1999). Brand Marketing. Beijing: Enterprise Management Press.

Ma, Dali. (2006).Clothing display technology. Beijing: China Textile Press.

Wang, Shouzhi. (2002). History of the world clothing. Beijing: China Youth Publishing House.

Jia, Jingsheng. (1999).Clothing color. Beijing: Higher Education Press. 